\title{
Principles of the mechanism of interfacial activation of a lipase: open and closed states and lipid - enzyme interactions in the limbus region of Candida antarctica Lipase B
}

\author{
M. Cianci \\ Department of Agricultural, Food and Environmental Sciences, Università Politecnica delle Marche, Via Brecce Bianche, 60131 \\ Ancona (Italy) \\ m.cianci@univpm.it
}

Lipases (E.C. 3.1.1.3) are ubiquitous hydrolases for the carboxyl ester bond of water-insoluble substrates such as triacylglycerols, phospholipids, and other insoluble substrates, acting in aqueous as well as in low-water media, thus being of considerable physiological significance with high interest also for their industrial applications. The hydrolysis reaction follows a two-step mechanism, or 'interfacial activation', with adsorption of the enzyme to a heterogeneous interface and subsequent enhancement of the lipolytic activity. Among lipases, Candida antarctica Lipase B (CALB) has never shown any significant interfacial activation, and a closed conformation of CALB has never been reported leading to the conclusion that its behaviour was due to the absence of a lid regulating the access to the active site. The lid open and closed conformations and their protonation states are observed in the crystal structure of CALB at $0.91 \AA$ resolution [1]. Having the open and closed states at atomic resolution allows relating protonation to the conformation, indicating the role of Asp145 and Lys290 in the conformation alteration. Once positioned within the catalytic triad, substrates are then hydrolysed, and products released. However, the intermediate steps of substrate transfer from the lipidic-aqueous phase to the enzyme surface and then down to the catalytic site are still unclear. By inhibiting CALB with ethyl phosphonate and incubating with glyceryl tributyrate (2,3-di(butanoyloxy)propyl butanoate), the crystal structure of the lipid-enzyme complex, at 1.55 $\AA$ resolution, shows the tributyrin in the limbus region of active site [2]. The substrate is found above the catalytic Ser, with the glycerol backbone readily pre-aligned for further processing by key interactions via an extended water network with $\alpha$-helix 10 and $\alpha$ helix5. These findings explain the lack of 'interfacial activation' of CALB and offer new elements to elucidate the mechanism of substrate recognition, transfer and catalysis of Candida antarctica Lipase B (CALB) and lipases in general.

[1] Stauch, B., Fisher, S. J., Cianci, M. (2015). Journal of Lipid Research, 56, 2348-2358.

[2] Silvestrini, L. \& Cianci, M. (2020). International Journal of Biological Macromolecules, 158, 358-363.

Keywords: Lipase; Fatty acid/metabolism; Lipids/chemistry; Enzymology/Enzyme regulation; ultra-high resolution; interfacial enzymology; tributyrin; CALB. 